NOTICE: this is the author's version of a work that was accepted for publication in International Journal of Psychophysiology. Changes resulting from the publishing process, such as peer review, editing, corrections, structural formatting, and other quality control mechanisms may not be reflected in this document. Changes may have been made to this work since it was submitted for publication. A definitive version was subsequently published in International Journal of

Psychophysiology, 92(3), doi: 10.1016/j.ijpsycho.2014.03.003

\title{
Knowledge of sequence structure prevents auditory distraction: An ERP study
}

\author{
Márta Volosin ${ }^{1}$ and János Horváth ${ }^{1}$ \\ ${ }^{1}$ Institute of Cognitive Neuroscience and Psychology Research Centre for Natural Sciences, \\ Hungarian Academy of Sciences, Budapest, Hungary.
}

E-mail addresses:

Márta Volosin: volosin.marta@ttk.mta.hu

János Horváth: horvath.janos@ttk.mta.hu

Address correspondence to:

Márta Volosin

Institute of Cognitive Neuroscience and Psychology, RCNS, HAS

Hungary, 1519 Budapest, P. O. Box: 286

e-mail address: volosin.marta@ttk.mta.hu

Phone number: +361 3826819

27 pages (including 2 figures) 


\begin{abstract}
Infrequent, salient stimuli often capture attention despite their task-irrelevancy, and disrupt on-going goal-directed behavior. A number of studies show that presenting cues signaling forthcoming deviants reduces distraction, which may be a "by-product" of cue-processing interference or the result of direct preparatory processes for the forthcoming distracter. In the present study, instead of "bursts" of cue information, information on the temporal structure of the stimulus sequence was provided. Young adults performed a spatial discrimination task where complex tones moving left or right were presented. In the predictable condition, every $7^{\text {th }}$ tone was a pitch-deviant, while in the random condition the position of deviants was random with a probability of $1 / 7$. Whereas the early event-related potential correlates of deviance-processing $(\mathrm{N} 1$, and $\mathrm{MMN})$ were unaffected by predictability, P3a amplitude was significantly reduced in the predictable condition, indicating that prevention of distraction was based on the knowledge about the temporal structure of the stimulus sequence.
\end{abstract}

Keywords: attention, distraction, ERP, P3a, prediction, regularity 


\section{Introduction}

Many tasks in our everyday life require the filtering of task-relevant and task irrelevant sensory events: Task-relevant events have to be processed as fast as possible, while taskirrelevant events should not consume processing resources at all. Such a "perfect" selective attention set, however, cannot be established: Unpredictable, rare stimuli easily capture our attention and disrupt the ongoing task-related behavior, that is, we get distracted. A number of studies show that the sensory system automatically responds to unpredictable, rare stimulus events (for a summary, see Escera et al., 2000), which may lead to involuntary allocation of attention to such events (Schröger, 1997). Recent studies show that when forthcoming, potentially distracting events are preceded by informative cues, the effects of distraction are reduced or eliminated (Sussman et al., 2003; Horváth et al., 2011; Horváth and Bendixen, 2012; Wetzel and Schröger, 2007; Wetzel et al., 2009, 2012). The goal of the present study was to investigate whether the prevention of distraction was also possible by providing information on forthcoming distracters without relying on explicit cues.

Cognitive processing related to distraction is usually investigated in oddball-paradigms, in which the presentation of frequent standard stimuli is interrupted by infrequent deviants. A variant of the oddball paradigm developed by Schröger and Wolff (1998b) allows unique insights into distraction-related processing. In this paradigm, long and short tones are presented equiprobably, and participants perform a duration discrimination task. Occasionally, randomly, the task-irrelevant tone pitch is changed (in about 10\% of the trials). For such deviants, prolonged response times, reduced hit rates and more false alarms were found than for standards.

Distraction effects can be found at the electrophysiological level as well: After deviance onset, a 
characteristic waveform can be observed in the deviant-minus-standard event-related potential (ERP) difference, starting with an enhanced N1 and mismatch negativity (MMN) at 100-250 ms, followed by a positivity at around $300 \mathrm{~ms}(\mathrm{P} 3 \mathrm{a})$, and finally a negative deflection occurs peaking around $500 \mathrm{~ms}$ (reorienting negativity - RON). The N1-effect and MMN reflect the activity of sensory change detection processes (Näätänen, 1982). P3a is generally assumed to reflect involuntary attention switching (Friedman et al., 2001; Polich, 2007), while RON is theorised to reflect the reorientation of attention to the original task (Schröger and Wolff, 1998a; Sussman et al., 2003). Similar results were found in auditory-visual paradigms in which targets were visual stimuli (e. g. odd or even numbers) and the distractors were sounds (Escera et al., 1998, 2000, 2001). Although the early studies using either auditory (Berti and Schröger, 2003; Schröger and Wolff, 1998a; Schröger and Wolff, 1998b) or auditory-visual (Escera et al., 1998, 2000, 2001) paradigms consistently found prolonged response times (RTs) and decreased accuracy, recent studies found abolished or even reversed behavioral effects (Li et al., 2013; Parmentier et al., 2010; SanMiguel, et al., 2010a; 2010b; Wetzel et al., 2012). These studies suggest that alerting and fore-period effects differ between standards and deviants, and these differences influence the behavioral results.

Interestingly, the paradigm can be also utilized to assess whether distraction can be prevented or reduced. Sussman et al. (2003) utilized the paradigm developed by Schröger and Wolff (1998b) but they presented visual cues before each tone. In the predictable condition, cues indicated whether the forthcoming tone was a standard or a pitch-deviant. In the unpredictable condition, the cues did not allow predicting whether the forthcoming tone was a standard or a deviant. In the unpredictable condition, the expected distraction effects were found: (delayed RTs to deviants in comparison to standards, and the elicitation of N1/MMN, P3a, and RON). In the 
predictable condition, however, the RT-delay, P3a and RON were abolished (predictability had no effect on the N1/MMN). These results were replicated in several studies using different experimental designs and manipulations of presentation (Horváth et al., 2011; Horváth and Bendixen, 2012; Wetzel and Schröger, 2007; Wetzel et al., 2009).

These studies showed that cues providing different degrees of predictability allow the reduction of distraction, but the mechanism behind the cuing effect is not fully understood yet. Although the prevalent interpretation of the cuing effect is that cues allow one to prepare for, and prevent distraction caused by deviants ("preparation"-hypothesis), other interpretations are also possible. The main alternative interpretation is that distraction-prevention is a "byproduct" of cue-processing: Because cues deliver information commensurate to that of the forthcoming deviant (i.e. their presentation frequencies are necessary the same, therefore deviant cues are deviants themselves within the cue sequence), processing this sudden "burst" of information may temporarily deplete processing resources, which in turn, may lead to reduced distraction effects. Direct evidence against the "byproduct"-hypothesis is scarce. There is only one study, conducted by Parmentier and Hebrero (2013), which showed that cues allowing the prediction of forthcoming deviants reduced distraction-related response-time delays even if the cues preceded the deviants by as much as $2250 \mathrm{~ms}$ (i.e. the reduction of RT-delay did not differ from that at 250 ms cue-tone separation). Because it seems unlikely that cue-related processing would block further processing for such a long time, this result supports the "preparation" account of the cuing effect.

The goal of the present study was to investigate distraction-prevention using the method of ERPs in a setting in which information on forthcoming distracters was not delivered in "bursts", but was available continuously. Investigating whether distraction can be reduced in this 
setting is important, because such an arrangement would allow the comparison of distractionprevention ability between groups potentially differing in their ability to process and utilize "bursts" of information. That is, the continuous availability of cue-information would eliminate confounds due to potential between-group cue-processing abilities. For example, if processing "burst"-like cues required $300 \mathrm{~ms}$ on average in one group, but required $500 \mathrm{~ms}$ in another, then cues appearing $400 \mathrm{~ms}$ before distracters would allow one group to fully prepare for the forthcoming distracters, while leaving the other group prone to their distracting effects. In this example, one would measure between-group differences in the efficiency in distractionprevention, but these differences would not reflect the ability to prevent distraction, rather, they would reflect a difference in cue information processing speed. Furthermore, even if the cuedistracter separation allowed both groups to process cue information in time, the utilization of this information depends on the willingness of participants to do so. The amount of effort needed to process cue information in the short time available may reduce the participants' motivation to utilize cue information at all (Horváth, 2013).

We administered an auditory distraction paradigm in which the presentation order of tones was either predictable (every $7^{\text {th }}$ tone was pitch-deviant) or random (with 1:6 deviant:standard ratio). The tones virtually moved either to the left or to the right and participants responded to the direction of the movement, ignoring sound frequency. As in previous studies, deviants in the predictable condition should be less distracting than those in the random condition because of the availability of information on forthcoming deviants. This arrangement, however, still provides a challenge: participants have to keep the current position within the sequence in mind to be able to prepare for forthcoming deviants. In order to minimize the effort needed, a visual counter showing the sequence position was presented as a constant reminder, which made information on 
forthcoming tones continuously available throughout the experimental blocks of the predictable condition. We hypothesized that knowledge about the stimulus sequence would reduce or abolish behavioral and ERP effects of distraction.

\section{Material and methods}

\subsection{Participants}

14 healthy young volunteers participated in the experiment ( 9 women, aged: 19-26 years, mean age: 22 years). All participants reported normal hearing and normal or corrected-to-normal vision. They received either modest financial compensation or course credit for participation, and gave written informed consent before the experiment, after the experimental procedures were explained to them.

\subsection{Materials and procedure}

Participants were sitting in a comfortable chair in a sound-attenuated room during the experiment. Each experimental block consisted of either random or predictable sequences of complex spatial sounds with 1300 ms SOA, through a Sennheiser (HD-600, Sennheiser, Wademark, Germany) headphone. The intensity of sounds was individually calibrated to $50 \mathrm{~dB}$ sensation level above the hearing threshold, determined by the method of limits.

Tones were generated with Csound version 5.7.11, using the head related transfer function tool "hrtfmove2" to simulate virtual movement. Due to a programming error, tones were generated with $44.1 \mathrm{kHz}$ sampling frequency, but replayed with $48.0 \mathrm{kHz}$, which did not 
substantially alter the perceived virtual movement. The frequency and velocity data values reported below correspond to what participants actually heard.

The duration of each tone was $643 \mathrm{~ms}$, with $9 \mathrm{~ms}$ rise and fall times. Each tone started on the virtual midline (they could be heard in both ears equally), then after $184 \mathrm{~ms}$ they moved $20^{\circ}$ toward the left or right (50-50\% probability) with constant angular velocity in $459 \mathrm{~ms}$, i.e. angular velocity was $43.54 \%$ sec. The tones were complex tones with six harmonics. The fundamental frequency of the tones was either $254 \mathrm{~Hz}$ (high) or $202 \mathrm{~Hz}$ (low). The amplitudes of the five harmonic overtones were $80 \%, 40 \%, 50 \%, 30 \%$ and $90 \%$ of the amplitude of the fundamental. Both frequencies could function as deviant $(14.28 \%)$ or standard $(85.71 \%)$. The role of frequencies (standard or deviant) was counterbalanced between participants: For seven participants, standards were high, for the other seven standards were low.

The participants' task was to indicate whether the tone moved to the left or to the right (regardless of its frequency), by pressing the key held in their corresponding hand. The instruction was to respond as fast and accurately as possible, immediately when the direction of the virtual movement could be assessed (without waiting for the sound-offset). Participants were informed before each block whether the presentation of the block was predictable or random. Each block consisted of frequent standard and rare deviant stimuli, presented with a 6:1 ratio. Thus, 154 tones were presented in each block (132 standards and 22 deviants). In predictable blocks every $7^{\text {th }}$ tone was deviant, in random blocks the tone order was randomized while keeping the 6:1 standard:deviant ratio.

To support keeping the current sequence position (and the forthcoming deviant tone) in mind, a visual counter was presented on a screen. Black digits from 1 to 7 were presented 
continuously in linear order in the middle of the gray screen, under a viewing angle of about $7^{\circ}$. The transition between digits occurred $44 \mathrm{~ms}$ before each tone. In the random condition where the order of the standards and deviants was completely unpredictable, the digits and their transition gave information only about the onset of the forthcoming tone (i. e., that it will be presented in $44 \mathrm{~ms}$ ) but not about its pitch (deviant or standard). In contrast, in the predictable condition, one of the transitions indicated that the next will be a deviant. The transition that was followed by the deviant was varied randomly between the participants (e.g., for some participants, the 1-to-2 transition was followed by a deviant, for others it was the 4-to-5, etc). Participants were explicitly told which transition was followed by the deviant. Each block started with a so-called "reminder" sequence, that is, the first four tones were presented in alternating order to the left and right directions, in order to make the direction discrimination easier. These four tones were not included in the analyses.

Estimating the effect of predictability is not trivial. Because in the predictable condition every $7^{\text {th }}$ stimulus was a deviant, one could select micro-sequences ending with a deviant and preceded by exactly six standards (but not seven, that is, the micro-sequence should end but also start with a deviant) from the random condition as a comparison. However, the proportion of such sequences is quite small. Therefore we included deviants preceded exactly by 4, 5, 7 or 8 standards as well. It is well-known that deviance-related processing activity is stronger when the deviant follows a longer regular stimulus sequence (Bendixen et al., 2007; Horváth et al., 2008; Sams et al., 1983; Winkler et al., 1996). Furthermore it is plausible that the activity-increase in deviance-related processing brought about by adding further stimuli to the preceding regular sequence decreases with each addition: For example, the activity increase when a deviant is preceded by 5 instead 4 standards is larger, or at least not smaller, in comparison to when it is 
preceded by 6 instead of 5. Because of this, adding the deviants preceded by 4-, 5-, 7- and 8standard micro-sequences results in less (or at least equal) deviance-related activity on average than for the deviants preceded by a 6-standard micro-sequence. That is, this selection of deviants results in an underestimation of the distraction-effects. Furthermore, in a random sequence, the frequency of micro-sequences with deviants preceded by a given number of standards decreases as the function of the number of preceding standards (e.g. there are less deviants preceded by exactly 5 standards than that preceded exactly by 4), which results in an even more conservative estimate (because more deviants with shorter preceding standard-micro-sequences are included in the average). Because we only included deviants preceded by 4-5-6-7-8 standards in the random condition, in order to achieve a similar number of trials as in the in predictable condition, about 2.5 times more random blocks were needed. Therefore, the experiment consisted of 5 predictable and 13 random blocks. The order of blocks was randomized with the constraint that predictable blocks could never immediately follow each other. The approximately 3.5 minutes-long blocks were separated by 1-2 minute long breaks, depending on the participant's preferences, with a longer, 10-15 minute break after 9 blocks. After each block, feedback on behavioral performance was given, which consisted of correct response rates, average response time and a distribution plot of correct response times within the block.

Before administering the experiment, the participants were familiarized with the task: Two practice blocks were presented, one before mounting the electrodes and one when the electrodes were already mounted. The practice blocks were about 3.5 minutes long and consisted of either only low or only high tones. 


\subsection{EEG recording}

The EEG was recorded with a sampling rate of $500 \mathrm{~Hz}$ with a Neuroscan Synamp 2 (Compumedics Inc., Victoria, Australia) amplifier, from $63 \mathrm{Ag} / \mathrm{AgCl}$ electrodes mounted on an EasyCap (EASYCAP GmbH, Herrsching, Germany) arranged according to the 10\% system (Nuwer et al., 1998), and filtered online with a $100 \mathrm{~Hz}$ lowpass filter. The reference electrode was placed on the tip of the nose, the ground electrode on the forehead. Horizontal electro-oculogram was measured from two electrodes placed at the outer canthi of the eyes. Vertical electrooculogram was calculated off-line as the difference between the signals of the Fp2 and an electrode under the right eye.

The EEG data were filtered offline, using a $20 \mathrm{~Hz}$ lowpass filter (Kaiser-windowed sinc finite impulse response filter, beta of 10.06, 1603 coefficients; $2 \mathrm{~Hz}$ transition bandwith, and stopband attenuation at least $100 \mathrm{~dB}$ ). $1344 \mathrm{~ms}$ long epochs were extracted from each trial, including a $144 \mathrm{~ms}$ pre-tone interval. Amplitude calculations were referred to the first $100 \mathrm{~ms}$ of the epochs (i.e. the interval before the visual stimulus transition). Epochs with a signal range exceeding $150 \mu \mathrm{V}$ on any channel, as well as the first four epochs of each experimental block ("reminder sequence") were discarded from the analyses. The epochs selected according to the preceding micro-sequences as described above were averaged separately by condition (predictable or random) and stimulus type (deviant or preceding standard), so predictable standard, predictable deviant, random standard and random deviant tones were included in the analyses.

\subsection{Statistical analysis}

For each participant medians of the response times were calculated, which gives more accurate results than means because of the skewed distribution of response times. Only correct 
responses between 300 and $1200 \mathrm{~ms}$ (following tone onset, i.e. between 100 and $1000 \mathrm{~ms}$ after the onset of virtual movement) were included in the response time analysis. $d$ ' sensitivity scores were calculated according to the signal detection theory (MacMillan and Creelman, 1991). Response times and d' were analyzed in repeated measures Condition $\times$ Stimulus analyses of variance (ANOVAs).

Distraction-related ERPs (N1/MMN and P3a) were identified in the group average deviant-minus-standard waveforms of the random condition. Individual ERP amplitudes were calculated in both conditions as the average signals in $40 \mathrm{~ms}$ long windows centered at peak latencies at the FCz electrode (where these ERPs typically peak in similar experiments, see e.g. Horváth et al., 2011; Horváth and Bendixen, 2012; Jankowiak and Berti, 2007). Additionally, and unexpectedly, two slow ERP waveforms were also observed, which were analyzed in $200 \mathrm{~ms}$ long windows. The amplitudes were submitted to Condition (random vs. predictable) $\times$ Stimulus (deviant vs. standard) repeated measures ANOVAs. Mean square error (MSE) and generalized effect sizes $\left(\eta_{G}^{2}\right)$ are reported (Bakeman, 2005; Olejnik and Algina, 2003).

\section{Results}

\subsection{Behavioral performance}

Neither the analyses of d' nor that of response times showed significant effects. The group-mean response time in the predictable condition was $576 \mathrm{~ms}$ in standard (standard deviation, $\mathrm{SD}=50 \mathrm{~ms}$ ) and $579 \mathrm{~ms}$ in deviant trials $(\mathrm{SD}=55 \mathrm{~ms})$, while in the random condition $577 \mathrm{~ms}$ was the average speed on standards $(\mathrm{SD}=49 \mathrm{~ms})$ and $578 \mathrm{~ms}$ on deviants $(\mathrm{SD}=59 \mathrm{~ms})$. These response times are referred to the onset of the tones (and not the time point the virtual movement started). Neither the main effect of Condition $(F[1,13]<.001, p=.99, \operatorname{MSE}<0.001$, 
$\left.\eta^{2}{ }_{\mathrm{G}}<.001\right)$, nor the main effect of Stimulus: standard or deviant $(\mathrm{F}[1,13]=.015, \mathrm{p}=0.7, \mathrm{MSE}<.001$, $\eta_{{ }_{\mathrm{G}}}^{2}<.0001$ ) was significant; and the Condition $\times$ Stimulus interaction did not show any significant effect either: $\left(\mathrm{F}[1,13]=.133, \mathrm{p}=.133\right.$, MSE $\left.<.001, \eta^{2}{ }_{\mathrm{G}}<.001\right)$. Regarding sensitivity, the mean of d'$\mathrm{s}$ in the predictable condition was 2.91 for standards $(\mathrm{SD}=0.65)$ and 2.8 for deviants $(\mathrm{SD}=0.58)$.

In the random condition, the mean of d'-s was 2.98 for standards $(\mathrm{SD}=0.79)$ and 2.85 for deviants $(\mathrm{SD}=0.73)$. No significant effect were found (Condition main effect: $\mathrm{F}[1,13]=.22, \mathrm{p}=.65$, $\mathrm{MSE}=.183, \eta_{\mathrm{G}}{ }_{\mathrm{G}}=.002$, Stimulus main effect: $\mathrm{F}[1,13]=.72, \mathrm{p}=.408, \mathrm{MSE}=.287, \eta^{2}{ }_{\mathrm{G}}=.008$, Condition $\times$ Stimulus interaction: $\left.\mathrm{F}[1,13]=.011, \mathrm{p}=.916, \mathrm{MSE}=.034, \eta^{2}{ }_{\mathrm{G}}<.001\right)$.

\subsection{ERPs}

After excluding artifact-contaminated epochs, individual ERPs were averaged for 88 deviants in the predictable condition (SD: 13.6); for 65 deviants in the random condition (SD: 13.44); 81.5 standards in the predictable condition (SD: 12.19) and 64.6 standards in the random condition (SD: 14.36). The group-average ERPs elicited at midline electrodes in the two types of trials and conditions, and corresponding deviant-minus-standard waveforms are presented in Fig. 1.

The ERP waveforms at $\mathrm{FCz}$ showed a negative-going trend before tone onset suggesting preparatory activity for the forthcoming tone. Tones elicited an N1 and a P2, which was followed by a negativity between 200 and $300 \mathrm{~ms}$ and a negative sustained activity of duration comparable to that of the tone. For deviants, the second negativity was overlapped by a positive waveform, and the sustained negativity persisted longer than for standards. This suggests that participants probably kept their attention slightly longer on deviants than on standards. The deviant-minusstandard difference waveform in the random condition showed an early negative difference (N1effect/MMN/N2b) with two negative peaks at $100 \mathrm{~ms}$ and $162 \mathrm{~ms}$, and a fronto-central P3a 
peaking at $286 \mathrm{~ms}$. In parallel with the differential fronto-central negativity resulting from the persistence of the sustained negativity for deviants, the difference waveform also showed a slow positive activity after $500 \mathrm{~ms}$, peaking at $634 \mathrm{~ms}$ on the $\mathrm{POz}$ lead in the random condition (identifiable as a P3b).

The ANOVA of the amplitudes at the first peak of the early negativity showed a significant Stimulus main effect: $\mathrm{F}(1,13)=39.766, \mathrm{p}<.001, \mathrm{MSE}=1.667, \eta_{\mathrm{G}}^{2}=.102$, indicating larger (more negative) N1 (and possibly MMN) amplitudes. Neither the Condition main effect $\left(\mathrm{F}[1,13]=2.14, \mathrm{p}=.16, \mathrm{MSE}=1.69, \eta_{\mathrm{G}}^{2}=.006\right.$ nor the Stimulus $\times$ Condition interaction $(\mathrm{F}[1,13]$ $\left.=.019, \mathrm{p}=.89, \mathrm{MSE}=2.629, \eta_{\mathrm{G}}{ }_{\mathrm{G}}<.001\right)$ was significant. For the second peak only a marginal Stimulus main effect was found: $F(1,13)=3.75, p=.075$, MSE $=6.53, \eta^{2}{ }_{G}=.034$. Neither the main effect of Condition: $\left(\mathrm{F}[1,13]=.75, \mathrm{p}=.4, \mathrm{MSE}=5.53, \eta_{\mathrm{G}}^{2}=.006\right)$, nor the interaction of Stimulus $\times$ Condition $\left(F[1,13]=1.02, p=.32, \mathrm{MSE}=3.048, \eta_{\mathrm{G}}{ }_{\mathrm{G}}=.004\right)$ reached statistical significance. The ANOVA of the amplitudes in the P3a latency-range showed a significant Stimulus main effect: $\mathrm{F}(1,13)=25.05, \mathrm{p}<.001, \mathrm{MSE}=22.15, \eta_{\mathrm{G}}^{2}=.35$ and a Condition $\times$ Stimulus interaction: $\mathrm{F}(1,13)=8.20, \mathrm{p}=.013, \mathrm{MSE}=2.04, \eta_{\mathrm{G}}^{2}=.016$, showing that $\mathrm{P} 3 \mathrm{a}$ amplitude was smaller in the predictable than in the random condition. A significant Condition main effect was not found: $\mathrm{F}(1,13)=.095, \mathrm{p}=.76, \mathrm{MSE}=4.52, \eta_{\mathrm{G}}{ }_{\mathrm{G}}<.001$. The topography of the P3a in the two conditions, and the modulatory P3a-effect (the difference of the deviant-minus-standard differences) are presented in Fig. 2. The ANOVA of the P3b activity on POz lead showed significant stimulus effect: $\mathrm{F}(1,13)=30.366, \mathrm{p}<.001, \mathrm{MSE}=3.837, \eta_{\mathrm{G}}^{2}=.053$, indicating that deviants evoked larger positive responses than standards. Neither the main effect of Condition $(F[1,13]=.008, p=.92$, MSE $\left.=8.813, \eta^{2}{ }_{\mathrm{G}}<.001\right)$ nor the Stimulus $\times$ Condition interaction was significant $(\mathrm{F}[1,13]=1.64$ $\left.\mathrm{p}=.22, \mathrm{MSE}=8.033, \eta_{\mathrm{G}}{ }_{\mathrm{G}}=.014\right)$. The ANOVA of the negative difference on AFz electrode showed 
a stimulus main effect: $\mathrm{F}(1,13)=4.80, \mathrm{p}=.047, \mathrm{MSE}=8.495, \eta_{\mathrm{G}}^{2}=.028$, indicating that amplitudes

for deviant tones were more negative than for standards. No significance was present regarding the Condition main effect $\left(\mathrm{F}[1,13]=.69, \mathrm{p}=.42, \mathrm{MSE}=8.654, \eta^{2}{ }_{\mathrm{G}}=.028\right)$ and the Stimulus $\times$ Condition interaction $\left(\mathrm{F}[1,13]=.92, \mathrm{p}=.76, \mathrm{MSE}=6.37, \eta_{\mathrm{G}}{ }<.001\right)$.

\section{Discussion}

The present study introduced an oddball paradigm in which the prevention of distraction was supported by the constant availability of information on the temporal structure of the stimulus sequence instead of supplying information on forthcoming distracters "in bursts" shortly before the distracter was presented. The results generally fit current views on distraction-related processing. Deviants elicited an N1-effect/MMN, P3a, a longer sustained frontal negativity, and a parietal P3b in comparison to standards preceding them. Importantly, P3a amplitude was significantly reduced in the predictable condition, despite using a conservative estimate of this effect. This supports the "preparation"-hypothesis, that is, this suggests that information on forthcoming deviants allowed participants to reduce the effects of distraction. The results also show that the present stimulation arrangement, which was designed to allow the elimination of confounds stemming from (between-group) differences in cue-processing abilities, is a viable alternative to the typically used cueing arrangements.

Although at first sight, the absence of distraction-related behavioral effects may seem to contradict the assumption that distraction had happened, it has to be kept in mind that behavioral responses in such paradigms are influenced by a number of factors (for example, differences in stimulus-triggered arousal level changes), which, in sum, may lead to reduced, but also to enhanced performance in a given paradigm (Li et al., 2013; Parmentier et al., 2010; SanMiguel et 
al., 2010a, 2010b; Wetzel et al., 2012). It is also possible that the lack of a significant behavioral distraction-effect is partially due to the "natural" stimulus-response mapping (congruent stimulusand response side), which may allow for a faster response, and less interference than in studies with arbitrary mappings (e.g. duration discrimination). Note that although behavioral distraction effects have been observed in previous studies using a laterality-based discrimination task (Wetzel et al., 2009), in these studies distracters were unique, spectrally rich, novel sounds (i.e. highly deviant sounds, differing in a number of features from standards), while in the present study deviants differed only in their pitch from standards.

Although P3a was significantly reduced in the predictable condition, the N1-effect/MMN was not similarly modulated by predictability. This is in consonance with previous studies, in which cue-information on forthcoming distracters did not affect the N1-effect/MMN (Horváth et al., 2011; Horváth and Bendixen, 2012; Wetzel and Schröger, 2007; Wetzel et al., 2011). In contrast with these studies, however, in the present study there was a definite theoretical possibility that knowledge of the sequence structure could lead to MMN reduction. It has been demonstrated that that under the right circumstances the deviant-detection system underlying MMN elicitation can represent regularities similar to that in the present study, and therefore, it may not respond to predictable deviants: Sussman et al. (1998) showed that in a passive arrangement (i.e. participants were reading during tone presentation), in a tone sequence in which every fifth tone was a deviant, deviants did not elicit an MMN, presumably, because the unit of representation was not the single tone, but the five-tone micro-sequence. The lack of MMN elicitation depended, however, on the rate of presentation: MMN was not present when the onsetto-onset interval (stimulus onset asynchrony - SOA) was $100 \mathrm{~ms}$, but a clear MMN was elicited when it was $1300 \mathrm{~ms}$ (see also Scherg et al., 1989). In a further study (Sussman et al., 2002), in 
which tones were presented with an SOA of $1000 \mathrm{~ms}$, and participants actively monitored whether the repeating five-tone-pattern was violated, no significant MMN was observed. Importantly, a significant MMN was, however, present in a condition in which participants actively monitored the sequence, but were unaware of the five-tone repeating structure. Similarly, significant MMNs were found in the study by Jankowiak and Berti (2007), in which deviants were presented at regular sequence positions (SOA of $2500 \mathrm{~ms}$ ) and participants were unaware of the regularity. This set of results suggests that top-down processes may influence the regularity representation underlying MMN elicitation even at a 1/s presentation rate, but the presence of the regularity in itself is insufficient to influence the MMN-mechanism. Based on these findings, one might expect that MMN would be reduced in the predictable condition of the present study, however, no such effect was found. The lack of modulation may be due to a number of differences between the paradigms: First, we used a relatively long SOA (1.3s vs. $1.0 \mathrm{~s})$ and micro-sequence length (every $7^{\text {th }}$ was a deviant vs. every $5^{\text {th }}$ was a deviant). Second, also, in contrast with Sussman et al.'s (2002) study, in which the feature defining the micro-sequence pattern and the task-relevant feature was the same (pitch), in our study the two features were different (pitch and lateral movement). Third, in Sussman et al.'s (2002) study the task was to detect a highly infrequent (2\%) pitch variant (which required a response only rarely), whereas in the present study the task was a $50-50 \%$ discrimination (which required a response on each trial).

The reduction of $\mathrm{P} 3 \mathrm{a}$ in the predictable condition indicates that knowledge about the stimulus sequence allowed the reduction of distraction. This result fits previous studies which used cues preceding deviants to prevent distraction (Horváth et al., 2011; Horváth and Bendixen, 2012; Sussman et al., 2003; Wetzel and Schröger, 2007; Wetzel et al., 2009). Because information on the tone sequence and forthcoming deviants was constantly available during stimulation, a 
"burst"-like cue processing was not required. This supports the notion that distraction is actively prevented by the use of predictive information, and the prevention is not (solely) a by-product of cue processing. In summary, the present study showed that knowledge of the stimulus sequence allowed one to prevent distraction as reflected by the P3a. This result is compatible with the notion that distraction can be prevented when information is available on forthcoming distracters. Moreover, the constant availability of information on forthcoming distracters eliminates potential processing bottlenecks inherent in other cue presentation based procedures. Therefore, the present stimulation arrangement may be more suitable to assess between-group differences in the ability to prevent distraction. 


\section{Acknowledgements}

The study was supported by the Hungarian Scientific Research Fund - OTKA (104635) and the János Bolyai Research Scholarship of the Hungarian Academy of Sciences (János Horváth). 


\section{References}

Bakeman, R., 2005. Recommended effect size statistics for repeated measures designs. Behavior Research Methods, 37(3), 379-384. doi: 10.3758/BF03192707

Bendixen, A., Roeber, U., Schröger, E., 2007. Regularity extraction and application in dynamic auditory stimulus sequences. J. Cogn. Neurosci. 19(10), 1664-1677. doi: 10.1162/jocn.2007.19.10.1664

Escera, C., Alho, K., Winkler I., Näätänen, R., 1998. Neural mechanisms of involuntary attention to acoustic novelty and change. J. Cogn. Neurosci. 10(5), 590-604. doi:10.1162/089892998562997

Escera, C., Alho, K., Schröger, E., Winkler, I., 2000. Involuntary attention and distractibility as evaluated with event-related brain potentials. Audiol. Neuro-Otol. 5, 151-166. doi: $10.1159 / 000013877$

Escera, C., Yago, E., Alho, K., 2001. Electrical responses reveal the temporal dynamics of brain events during involuntary attention switching. Eur. J. Neurosci. 14, 877-883. doi: 10.1046/j.0953-816x.2001.01707.x

Friedman, D., Cycowitz, Y.M., Gatea, H., 2001. The novelty P3: an event-related potential (ERP) sign of the brain's evaluation of novelty. Neurosci. Behav. Rev. 25, 355-373. doi: $10.1016 / \mathrm{S} 0149-7634(01) 00019-7$

Horváth, J., 2013. Preparation interval and cue-utilization in the prevention of distraction. Exp. Brain Res. 231, 179-190. doi: 10.1007/s00221-013-3681-3 
Horváth, J., Bendixen, A., 2012. Preventing distraction by probabilistic cueing. Int. J. Psychophysiol. 83, 342-347. doi: 10.1016/j.ijpsycho.2011.11.019

Horváth, J., Sussman, E., Winkler, I., Schröger, E., 2011. Preventing distraction: Assessing stimulus-specific and general effects of the predictive cueing of deviant auditory events. Biol. Psychol. 87, 35-48. doi: 10.1016/j.biopsycho.2011.01.011

Horváth, J., Winkler, I., Bendixen, A., 2008. Do N1/MMN, P3a and RON form a strongly coupled chain reflecting the three stages of auditory distraction? Biol. Psychol. 79, 139147. doi: 10.1016/j.biopsycho.2008.04.001

Jankowiak, A., Berti, S., 2007. Behavioral and event-related potential distraction effects with regularly occurring auditory deviants. Psychophysiol. 44, 79-85. doi: 10.1111/j.14698986.2006.00479.x

Li, B., Parmentier, F.B.R., Zhang, M., 2013. Behavioral distraction by auditory deviance is mediated by the sound's informational value: Evidence from an auditory discrimination task. Exp. Psychol. 60(4), 260-268. doi: 10.1027/1618-3169/a000196

MacMillan, N., Creelman, C., 1991. Detection Theory: A User's Guide. Cambridge University Press, Cambridge.

Näätanen, R., 1982. Processing Negativity: An evoked-potential reflection on selective attention. Psychol. Bull. 92(3), 605-640. doi: 10.1037/0033-2909.92.3.605

Nuwer, M., Comi, G., Emerson, R., Fuglsang-Frederiksen, A., Guérit, J-M., Hinrichs, H., Ikeda, A., Luccas, F.J.C., Rappelsburger, P., 1998. IFCN standards for digital recording of 
clinical EEG. Electroencephalogr. Clin. Neurophysiol. 106, 259-261. doi: 10.1016/S00134694(97)00106-5

Olejnik, S., Algina, J., 2003. Generalized Eta and Omega Squared Statistics: Measures of Effect Size for Some Common Research Designs. Psychological Methods, 8(4), 434-447. doi: 10.1037/1082-989X.8.4.434

Parmentier, F.B.R., Elsley, J.V., Ljungberg, K., 2010. Behavioral distraction by auditory novelty: The role of distracter's informational value. Cogn. 115, 504-511. doi: 10.1016/j.cognition.2010.03.002

Parmentier, F.B.R., Hebrero, M., 2013. Cognitive control of involuntary distraction by deviant sounds. J. Exp. Psychol.: Learning, Mem., Cogn. 39, 1635-1641. doi: 10.1037/a0032421.

Polich, J., 2007. Updating P300: An integrative theory of P3a and P3b. Clin. Neuropsychol. 118(10), 2128-2148. doi: 10.1016/j.clinph.2007.04.019

Sams, M., Alho, K., Näätänen, R., 1983. Sequential effects on the ERP in discriminating two stimuli. Biol. Psychol. 17, 41-58. doi: 10.1016/0301-0511(83)90065-0

SanMiguel, I., Linden, D., Escera, C., 2010a. Attention capture by novel sounds: Distraction versus facilitation. Eur. J. Cogn. Psych. 22(4), 481-515. doi: $10.1080 / 09541440902930994$

SanMiguel, I., Morgan, H.M., Klein, C., Linden, D., Escera, C., 2010b. On the functional significance of Novelty-P3. Facilitation by unexpected novel sounds. Biol. Psychol. 83, 143-152. doi: 10.1016/j.biopsycho.2009.11.012 
Scherg, M., Vajsar, J., Picton, T.W., 1989. A source analysis of the late human auditory evoked potentials. J. Cogn. Neurosci. 1(4), 336-355. doi: 10.1162/jocn.1989.1.4.336

Schröger, E., 1997. On the detection of auditory deviations: A pre-attentive activation model. Psychophysiol. 34(3), 245-257. doi: 10.1111/j.1469-8986.1997.tb02395.x

Schröger, E., Wolff, C., 1998a. Attentional orienting and reorienting is indicated by human eventrelated brain potentials. NeuroRep. 9(15), 3355-3358. doi: 10.1097/00001756199810260-00003.

Schröger, E., Wolff, C., 1998b. Behavioral and electrophysiological effects of task-irrelevant sound change: a new distraction paradigm. Cogn. Brain Res. 7, 71-87. doi: 10.1016/S0926-6410(98)00013-5

Sussman, E., Ritter, W., Vaughan, H.G., 1998. Predictability of stimulus deviance and the mismatch negativity. NeuroRep. 9(18), 4167-4170. doi:10.1097/00001756-19981221000031

Sussman, E., Winkler, I., Huotilainen, M., Ritter, W., Näätänen, R., 2002. Top-down effects can modify the initially stimulus-driven auditory organization. Cogn. Brain Res. 13, 393-405. doi: 10.1016/S0926-6410(01)00131-8

Sussman, E., Winkler I., Schröger, E., 2003. Top-down control over involuntary attention switching in the auditory modality. Psychon. Bull. Rev. 10(3), 630-637. doi: 10.3758/BF03196525

Wetzel, N., Schröger, E., 2007. Cognitive control of involuntary attention and distraction in children and adolescents. Brain Res. 1155, 134-146. doi: 10.1016/j.brainres.2007.04.022 
Wetzel, N., Widmann, A., Schröger, E., 2009. The cognitive control of distraction by novelty in children aged 7-8 and adults. Psychophysiol. 46, 607-616. doi: 10.1111/j.14698986.2009.00789.x

Wetzel, N., Widmann, A., Schröger, E., 2012. Distraction and facilitation - Two faces of the same coin? J. Exp. Psychol.: Hum. Percept. Perform. 38(3), 664-674. doi: 10.1037/a0025856

Winkler, I., Cowan, N., Csépe, V., Czigler, I., Näätänen, R., 1996. Interactions between transient and long-term auditory memory as reflected by the mismatch negativity. J. Cogn. Neurosci. 8(5), 403-415. doi: 10.1162/jocn.1996.8.5.403 


\section{Figure captions}

\section{Figure 1}

Group-average $(\mathrm{N}=14)$ ERPs to deviants, and standards preceding them in the random (left) and the predictable (right) conditions, and the corresponding deviant-minus-standard difference waveforms (middle column) at selected midline and averaged mastoid leads.

\section{Figure 2}

Group-average $(\mathrm{N}=14)$ topographical distribution of the P3a in the random (left panel) and in the predictable condition (middle panel). The P3a-effect (right panel) is calculated as the betweencondition difference of the deviant-minus-standard ERP differences. 


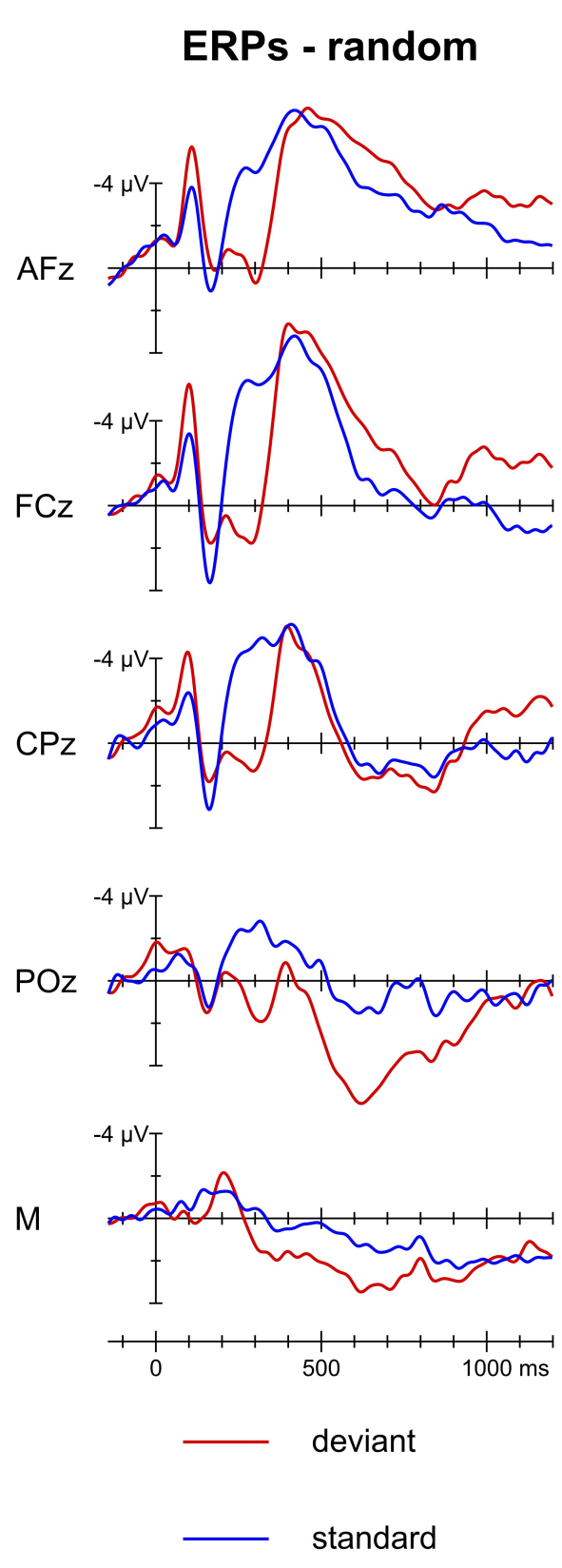

\section{Deviant minus standard difference waveforms}

\section{ERPs - predictable}
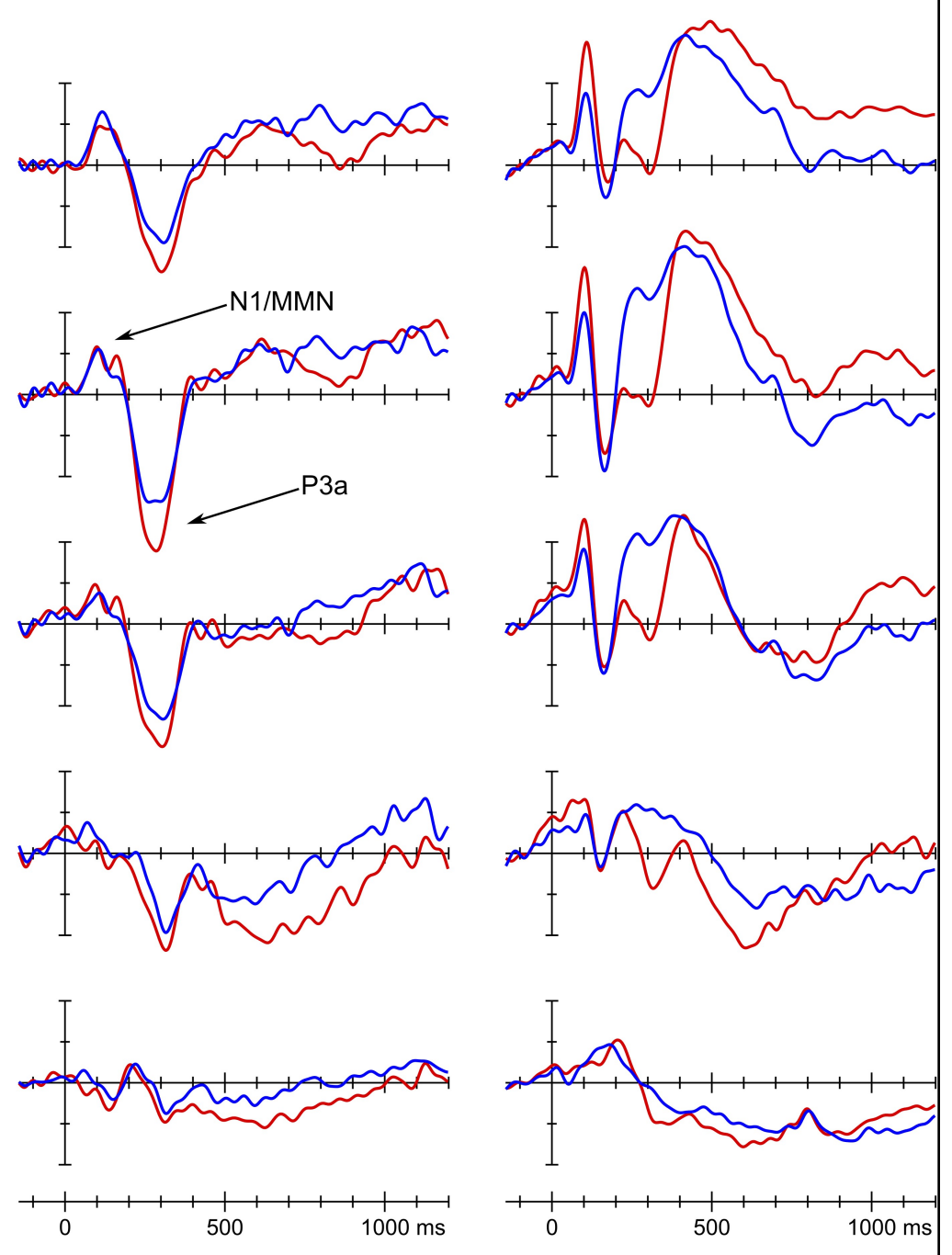

random deviant

minus random standard

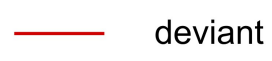

predictable deviant

minus predictable standard

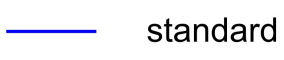

Figure 1. Group-average $(\mathrm{N}=14)$ ERPs to deviants, and standards preceding them in the random (left) and the predictable (right) conditions, and the corresponding deviant-minus-standard difference waveforms (middle column) at selected midline and averaged mastoid leads. 


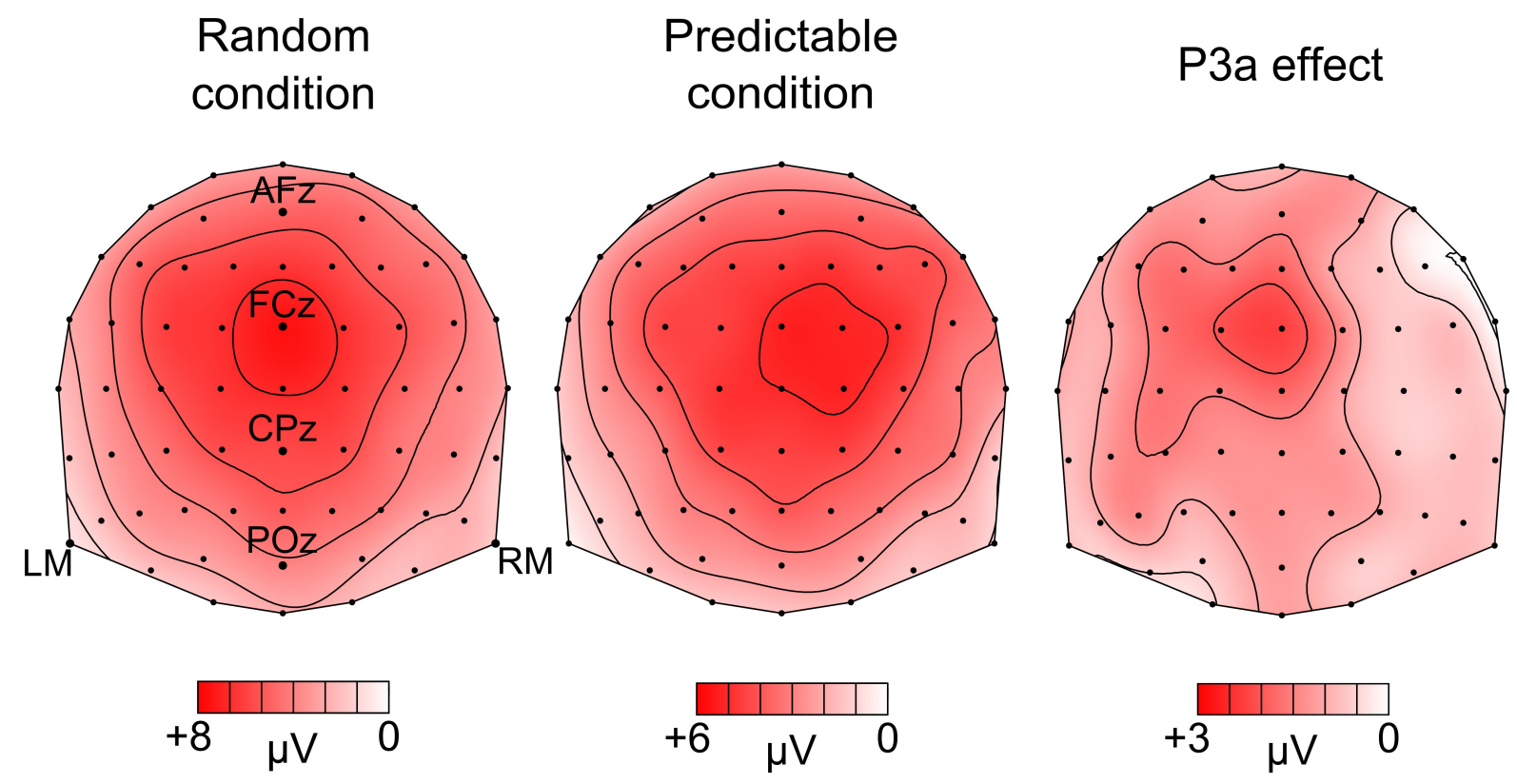

Figure 2. Group-average $(\mathrm{N}=14)$ topographical distribution of the $\mathrm{P} 3 \mathrm{a}$ in the random (left panel) and in the predictable condition (middle panel). The P3a-effect (right panel) is calculated as the between-condition difference of the deviant-minus-standard ERP differences. 